\title{
Topological surface wave metamaterials for robust vibration attenuation and energy harvesting
}

\author{
Xinyue $\mathrm{Wu}^{1}$, Yabin $\mathrm{Jin}^{2,{ }^{*}}$, Abdelkrim Khelif ${ }^{3}$, Xiaoying Zhuang ${ }^{1,4, *}$, Timon Rabczuk ${ }^{5}$, \\ Bahram Djafari-Rouhani $^{6}$ \\ ${ }^{1}$ Department of Geotechnical Engineering, College of Civil Engineering, Tongji University, \\ 200092 Shanghai, China \\ ${ }^{2}$ School of Aerospace Engineering and Applied Mechanics, Tongji University, 200092 Shanghai, \\ China \\ ${ }^{3}$ Institut FEMTO-ST, CNRS, Université de Bourgogne Franche-Comté,25030 Besançon, France \\ ${ }^{4}$ Institute of Photonics, Department of Mathematics and Physics, Leibniz University Hannover, \\ 30157 Hannover, Germany \\ ${ }^{5}$ Institute of Structural Mechanics, Bauhaus-Universität Weimar, Weimar, D-99423, Germany \\ ${ }^{6}$ Institut d'Electronique, de Microélectonique et de Nanotechnologie, UMR CNRS 8520, \\ Département de Physique, Université de Lille, 59650 Villeneuve d'Ascq, France \\ *Corresponding authors: 083623jinyabin@tongji.edu.cn; zhuang@iop.uni-hannover.de
}

\section{Abstract}

Metamaterials are extensively utilized to manipulate ground surface waves for vibration isolation within the bandgap frequency ranges whereas topological crystals allow the creation of robust edge states immune to scattering by defects. In this work, we propose a topological surface wave metamaterial working in the Hertz frequency range, constituted of triangle shape concrete pillars arranged in a honeycomb lattice and deposited on the soil ground. Based on the analogue of quantum valley Hall effect, a non-trivial bandgap is formed from the degeneracy lifting of the Dirac cone at the $\mathrm{K}$ point of the Brillouin zone by breaking the inversion symmetry of the two pillars in each unit cell. A topological interface is created between two different crystal phases and a topological edge state based on surface acoustic wave propagation is demonstrated. The robustness of the topologically protected edge state is quantitatively analyzed in presence of various defects and disorders. Finally, we take advantage of the robust and compact topological edge state for designing a harvesting energy device. The results demonstrate the functionality of the proposed structure for both robust 
surface vibration reduction as well as energy harvesting by designing proper topological waveguides.

Keywords: Surface wave metamaterial, topological insulator, vibration attenuation, energy harvesting, robustness.

\section{Introduction}

Mechanical vibrations in urban areas resulting from engineering construction and ground transportation may damage buildings and bridges, impact the operation of sophisticated instruments in high-tech laboratories and intrude residents' slumber. Apart from the airborne acoustic wave, the energy of these vibrations is generally concentrated in the depth of one wavelength underneath the ground surface, that is, primarily propagating in the form of surface waves, and the frequency range is usually below $20.0 \mathrm{~Hz}[1,2]$.

Mechanical metamaterials[3] whose configurations can be artificially fabricated and modulated have been proven to manipulate the propagation of mechanical waves with new effects such as wave isolation[4-9], topological insulator[10-19], Fano resonance[20-23], chirality[24], focusing and imaging[25], among others. Especially, surface wave metamaterials[1,26-36] are designed mainly for transmission suppression of low-frequency surface wave in certain frequency ranges based on the conception of hybridized bandgaps. Nonetheless, energy harvesting and signal detection of the subwavelength ground surface wave can be additional functions for surface wave metamaterials, remaining a meaningful and challenging issue that has hitherto received few attentions. It is possible to transform the surface wave into available electric energy and detect ground dynamics for potential catastrophe early warning.

For practical applications, defects and disturbances are ubiquitous in surface wave metamaterials, hence the surface wave control with high robustness is a crucial challenge. Recently, the rapid development of metamaterial topological insulator provides an efficient way to realize robust surface wave metamaterials. Topology provides a method to describe global wave properties over a band structure which is 
able to be conserved under certain local perturbations. In recent years, the development of topological insulators extended from condensed matter physics to classic wave systems such as acoustic and elastic waves. In general, Dirac cone dispersion results from the lattice symmetry which is protected by the space inversion and the timereversal symmetry. Different mechanisms like the quantum Hall, quantum spin Hall and valley Hall effects are proposed to open a bandgap from the Dirac cone[12]. According to the elastic analog of the quantum valley Hall effect, topological phases associated to opened bandgaps from the degeneracy lifting of the Dirac cone can be achieved via the inversion symmetry breaking [37]. When combining two bulk metamaterials with the opposite topological phases, topologically protected edge states can be induced showing strong energy localization at the interface within certain frequency ranges. The effect has been employed successfully in Lamb wave manipulation with significant robustness[37-43], revealing a great potential in the ground surface waves manipulation. For the application to surface acoustic waves, Wang et al. reported robust guiding valley-dependent edge states for Rayleigh waves excited by chiral sources at around $30 \mathrm{MHz}$ [44]; Topological chiral edge state is also realized on a periodically corrugated surface[45].

For ground surface waves in macro scale, industrial and traffic activities induced vibrations are different from chiral excitations. In this work, we study the topological protected edge modes based on surface acoustic waves within the non-trivial bandgap of a pillared metamaterial. The edge states are excited by a force point source with either out-of-plane or in plane polarization. Indeed, these are the main polarizations of the typical surface waves such as Rayleigh or Love waves. In Sec. 2, we describe the models and the implemented methods for designing non-trivial bandgaps and topological edge modes of the pillared metamaterial based on surface acoustic waves. In Sec. 3, we quantitatively study the robustness of the designed topological edge mode against three kinds of perturbations. In Sec. 4, we adopt the robust and compact topological edge mode whose path can be designed, according to the need, to study energy harvesting properties by using piezoelectric materials. Finally, a summary of the 
main results is presented in Sec. 5 .

\section{Models and Methods}

We propose a design constituted by a honeycomb lattice of equilateral triangular section pillars made of concrete on the surface of soil. We are interested by ground surface waves with frequency range under $20.0 \mathrm{~Hz}$, so we chose a lattice constant of $a$ $=2.0 \mathrm{~m}$ and the parameters of the pair of identical pillars in the unit cell are fixed as edge length $d=0.8 \mathrm{~m}$ and height $h=2.0 \mathrm{~m}$. The design and the unit cell for the 3D simulation model are shown in FIG. 1(a). To prevent the physical surface modes being perturbed by the unphysical Lamb type waves of the thick substrate plate in the simulation model, the depth of soil in the unit cell is chosen to be $H=30.0 \mathrm{~m}$ and a numerical low-reflecting boundary is applied at the bottom surface to avoid wave reflection. The soil (Young's modulus $E_{\mathrm{s}}=10 \mathrm{MPa}$, Possion's ratio $v_{\mathrm{s}}=0.3$, mass density $\rho_{\mathrm{s}}=1800 \mathrm{~kg} / \mathrm{m}^{3}$ ) and concrete pillars (Young's modulus $E_{\mathrm{c}}=40 \mathrm{GPa}$, Possion's ratio $v_{\mathrm{c}}=0.25$, mass density $\rho_{\mathrm{c}}=2500 \mathrm{~kg} / \mathrm{m}^{3}$ ) are assumed to be homogeneous, isotropic and linearly elastic materials[1]. Therefore, the equation of motions in solids can be written with harmonic time dependence assumption as

$$
\begin{gathered}
\omega^{2} \rho u_{i}=-\sigma_{i j, j} \\
\sigma_{i j}=c_{i j k l} u_{k, l}
\end{gathered}
$$

where $\omega$ is the angular frequency, $u_{i}$ the displacement component, $\sigma_{i j}$ the stress tensor and $c_{i j k l}$ the stiffness tensors that can be expressed as a function of the Young's modulus $E$ and Poisson ratio $v$. To obtain the dispersion relations, Bloch periodic conditions are applied on the four vertical surfaces of the unit cells based on Bloch theorem[46] as

$$
\boldsymbol{u}(\boldsymbol{r}+\boldsymbol{a})=\boldsymbol{u}(\boldsymbol{r}) e^{i \boldsymbol{k} \cdot \boldsymbol{a}}
$$

where $\boldsymbol{a}$ is the lattice vector, $\boldsymbol{r}$ the position vector, and $\boldsymbol{k}=\left[k_{x}, k_{y}\right]$ the wave vector. In addition, stress-free conditions are applied on the other top boundaries of the simulation model. We sweep the wave vector along the high-symmetry axes $\Gamma-\mathrm{K}-\mathrm{M}-\Gamma$ in the 
first irreducible Brillouin zone of the honeycomb lattice (see FIG. 1(c)) and search the eigenvalues to find dispersion relations with the help of the finite element method. It is worth mentioning that this work is dealing with the interaction between surface waves and pillars, thereby the vibrating modes are represented only under the sound cone of the substrate which means below the bulk shear wave[4, 47] (shown as the solid black lines in FIGs. 1(c)-(d)) where the propagation is prohibited towards the bulk. When the two pillars in the unit cell are identical as in FIG. 1(a), a Dirac cone based on surface waves dispersion curves emerges at $11.02 \mathrm{~Hz}$ at the K point in FIG. 1(c). The frequency of the Dirac cone is dependent on the pillar's resonant frequency[48]. Thus, the working frequency of the Dirac cone can be further tuned by designing the proper pillar's geometry.

Then, we decrease the edge length of one equilateral triangular pillar in the unit cell to $d_{1}=0.7 \mathrm{~m}$ while we increase that of the other to $d_{2}=1.0 \mathrm{~m}$, and keep unchanged the distance between the two triangle centroids (see FIG. 1(b)). Thereby, the inversion symmetry of the unit cell is broken while the $C_{3 \mathrm{v}}$ symmetry is maintained, which further induces a non-trivial bandgap in the range $[10.22,11.44] \mathrm{Hz}$. The degeneracy of the Dirac point is lifted and the limits of the bandgap at the $\mathrm{K}$ point occur at the points, $\mathrm{D}_{1}$ $(11.61 \mathrm{~Hz})$ and $\mathrm{D}_{2}(10.22 \mathrm{~Hz})$ with the inverted symmetry eigenmodes as shown in FIGs. 1(e)-(f). The elastic energy flux travels anticlockwise and clockwise around the pillars for $\mathrm{D}_{1}$ and $\mathrm{D}_{2}$ valleys, respectively. If $d_{1}=1.0 \mathrm{~m}$ and $d_{2}=0.7 \mathrm{~m}$, the chirality of the valley states for the lower and higher bands associated to the opened bandgap becomes inverted. Therefore, the valley Chern number associated with this lower and upper bands have opposite signs [16, 41], which further supports an elastic analog of quantum valley Hall effect. As a consequence, the opened bandgap becomes non-trivial. The wavelength of referenced Rayleigh wave propagating along the free surface without pillars at the center frequency of the non-trivial bandgap is about $4 \mathrm{~m}$, also demonstrating a subwavelength property of the pillared metamaterial. The induced nontrivial bandgap is able to provide robust surface wave attenuation. 
(a)

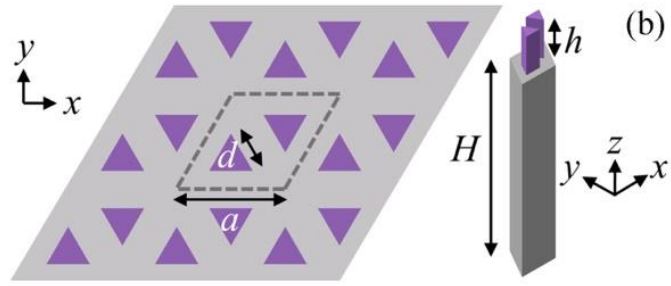

(b)

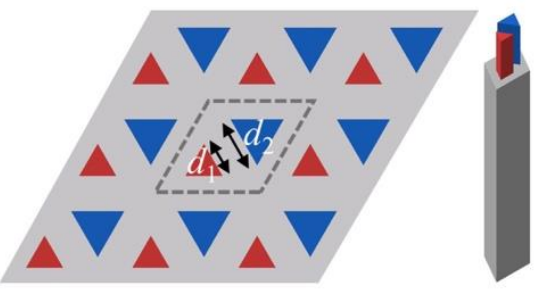

(d)

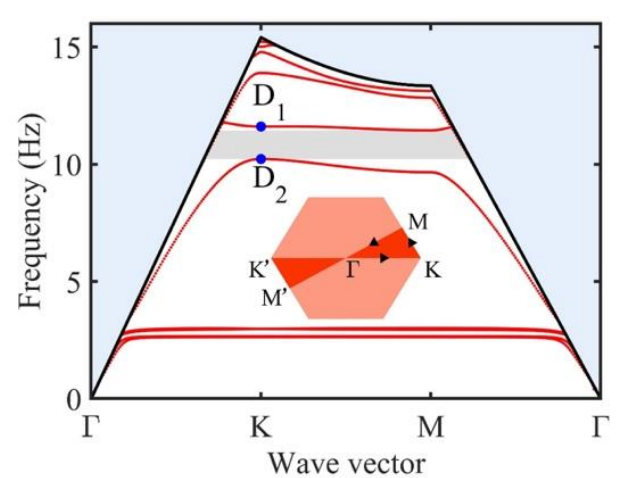

(f)

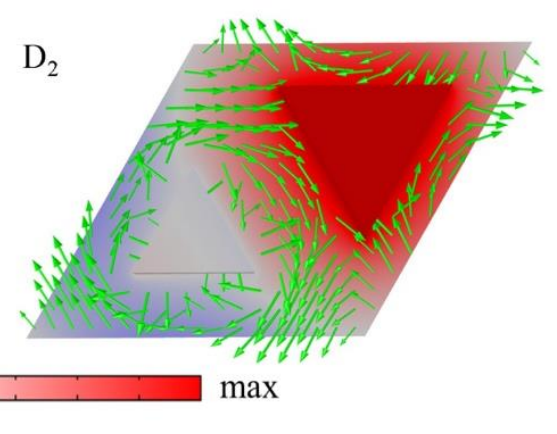

(c)

(e)

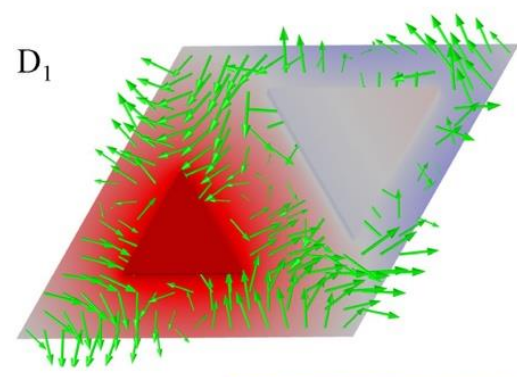

$-\max$

FIG. 1 (a) A honeycomb lattice with two identical triangular shape pillars in the unit cell. The soil below the pillars is represented by a thick (gray) substrate. (b) The honeycomb lattice with two different pillars in the unit cell breaking the inversion symmetry while preserving the $C_{3 \mathrm{v}}$ symmetry. (c) Dispersion curves of the surface waves (red lines) for the structure shown in (a) displaying a Dirac point (D point, $11.02 \mathrm{~Hz}$ ). The blue shaded area represents the sound cone. The light red hexagon and the crimson right triangle indicate the first Brillouin zone and the first irreducible Brillouin zone, respectively. (d) Dispersion curves of the surface waves (red lines) for the structure shown in (b) showing the lifting of the Dirac point degeneracy towards the points $\mathrm{D}_{1}(11.61 \mathrm{~Hz})$ and $\mathrm{D}_{2}(10.22 \mathrm{~Hz})$. The light blue and gray areas represent the propagating bands of the substrate and the bandgap within $[10.22,11.44] \mathrm{Hz}$, respectively. (e) and (f) The eigenmodes (real part of the out-of-plane displacement $u_{z}$ ) of $\mathrm{D}_{1}$ and $\mathrm{D}_{2}$. In the color bar, the red and blue colors refer to the positive and negative values of the real part of $u_{z}$, respectively. Elastic energy flux is shown as green arrows.

We turn now our attention to the design of a topological interface supporting an edge mode. Based on the unit cell shown in FIG. 1(b), we consider two types of topological interfaces by the juxtaposition of two crystals connected either by two large 
pillars (called L-L) or two small pillars (called S-S). We design two strips consisting of 20-unit cells with either an L-L or an S-S interface at the middle (FIGs. 2(c)-(d), respectively). Periodic and stress-free boundary conditions are respectively applied to the wider horizontal surfaces and to the narrower vertical surfaces. The detailed information can be found in Appendix A. We sweep the wave vector around $k_{x}=\pi / a$ along the $x$ direction, and calculate the dispersion diagram shown in FIGs. 2(a)-(b) for the L-L and the S-S types of interfaces, respectively. To detect the presence of an edge mode, localized in the vicinity of each interface, we propose a localization ratio $\beta$ defined as the ratio of the integrals of the kinetic energy density $\left(\kappa=\rho\left(\dot{u}_{x}^{2}+\dot{u}_{y}^{2}+\dot{u}_{z}^{2}\right) / 2\right)$ over the two pillars at the interface and over all the pillars. The localization ratio is expressed as

$$
\beta=\frac{\iiint_{\text {interface }} \kappa \mathrm{d} V}{\iiint_{\text {all }} \kappa \mathrm{d} V}
$$

A high value of $\beta$ indicates that the eigenmode is mostly concentrated at the interface, revealing an interface mode. In FIGs. 2(a)-(b), the dispersion diagrams display a bright edge mode around $10.86 \mathrm{~Hz}$ for the L-L type interface and within the interval [10.34, 10.45] Hz for the S-S type interface. The other branches appearing in the bandgap of surface waves result from the band folding of the bulk modes belonging to the soil. We also present the dominant out-of-plane displacement fields of the eigenmodes at the points A and B in FIGs. 2(c)-(d). One can clearly recognize edge modes localized around the interfaces, with a higher confinement for mode B as compared to mode A. The two pillars at the interface show a dipolar and a monopolar motion in FIGs. 2(c)(d), respectively. Since the edge modes based on surface acoustic waves are constructed within the non-trivial bandgap (shaded grey zone), they are expected to exhibit topological protected properties such as showing high robustness against certain perturbations and being free of backscattering. 
(a)

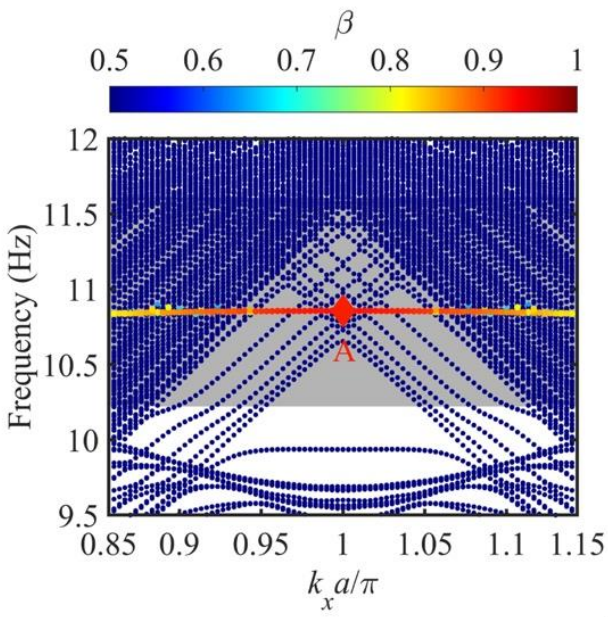

(b)

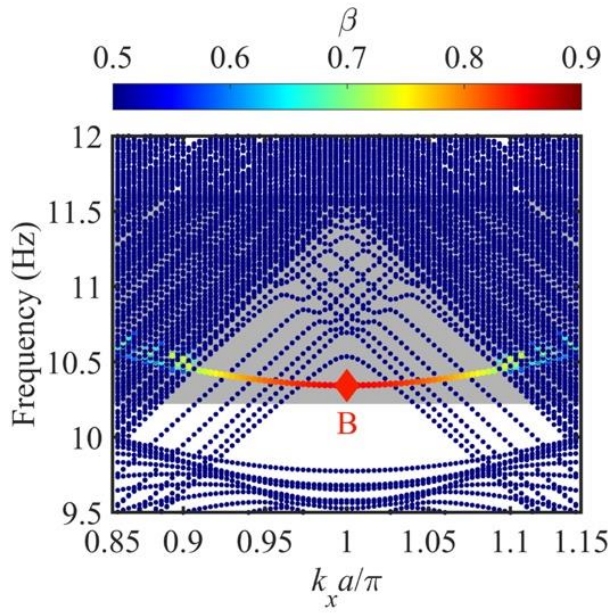

A: L-L

(c)

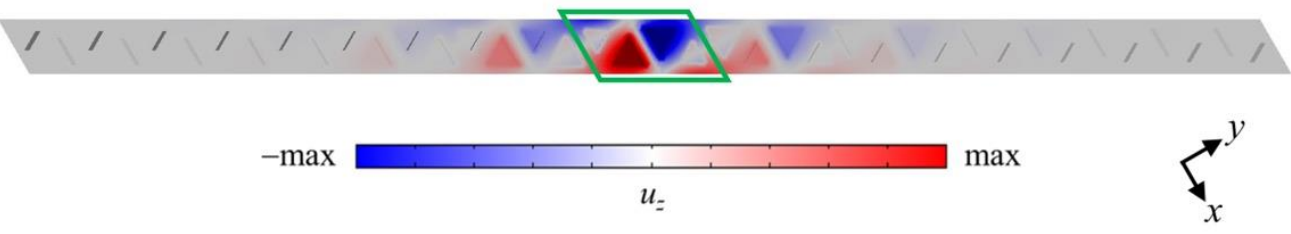

(d)

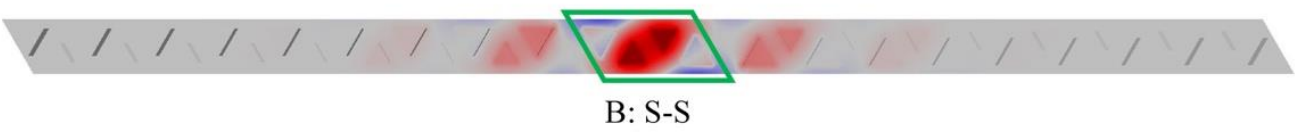

FIG. 2 Two strips are constructed by placing two topologically distinct lattices adjacently, each of them containing 10 unit cells and the interface at the middle being defined by two neighboring large (L-L) or small (S-S) pillars. (a) and (b) Dispersion diagrams for the L-L type and S-S type interfaces. The color represents the value of localization ratio $\beta$. The grey zones in (a) and (b) stand for the non-trivial bandgaps. (c) and (d) the out-of-plane displacement of the edge modes A and B in (a) and (b). The unit cells besides the interface are marked out by the green boxes. 
(a)

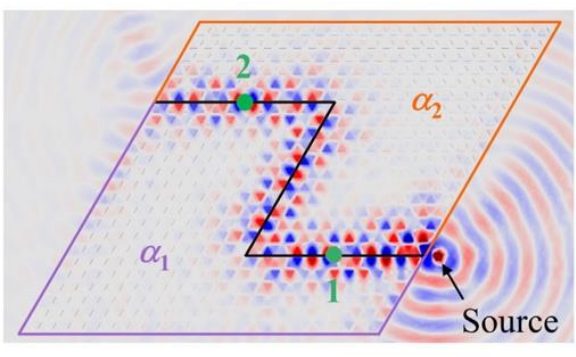

$-\max$

(c)

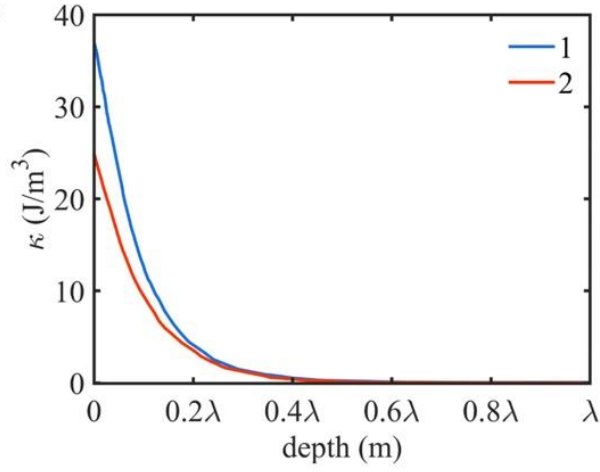

(e)

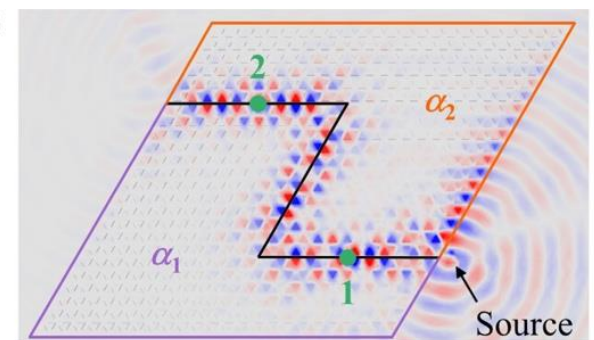

(b)

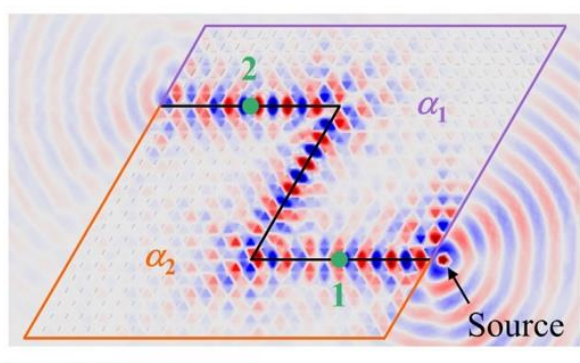

$\max$

$u_{z}$

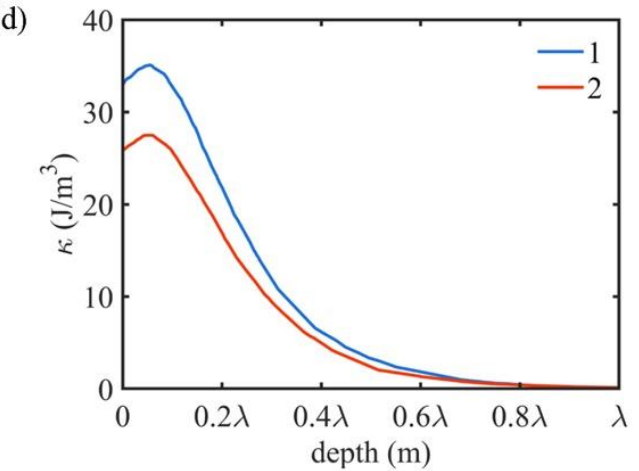

(f)

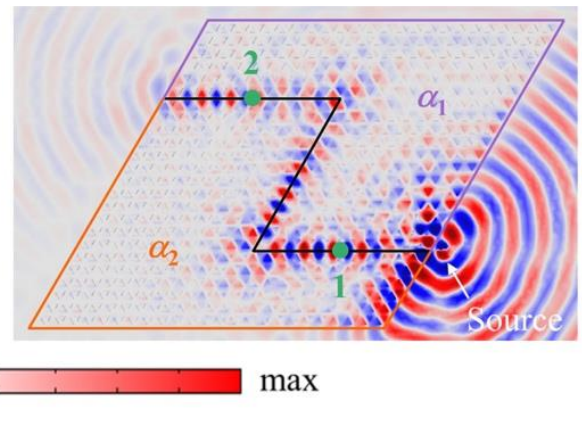

(h)

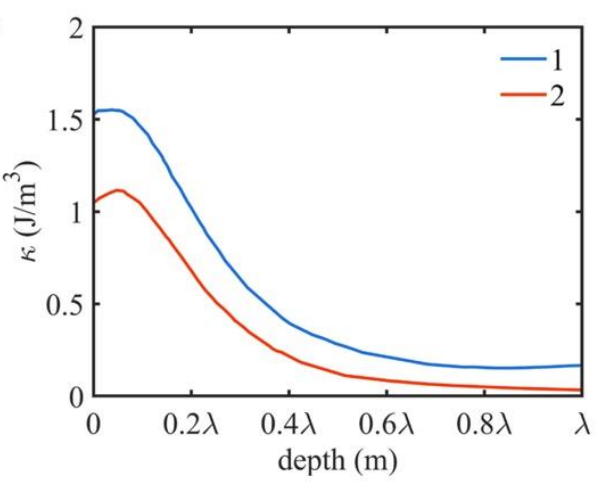

(g)

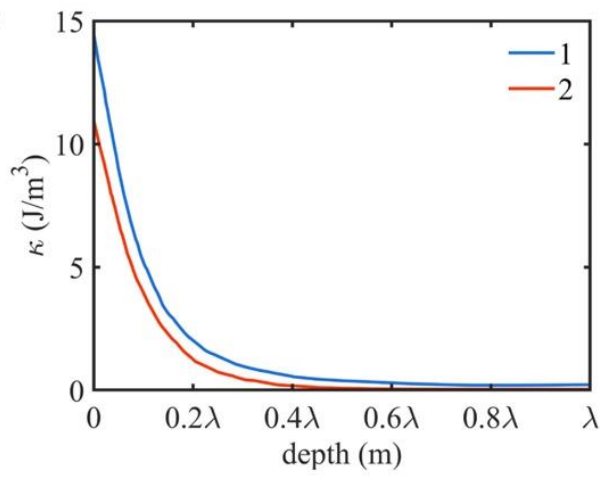

FIG. 3 Out-of-plane displacement fields with the out-of-plane point force excitation for the zig-zag waveguide (black curves) for (a) the L-L type and (b) the S-S type at $10.75 \mathrm{~Hz}$ and $10.40 \mathrm{~Hz}$, respectively. $\alpha_{1}$ and $\alpha_{2}$ refer to the two distinct topological phases. In (c) and (d), the diagrams of the kinetic energy density at the dots 1 and 2 along the soil depth are plotted for the two types. Panels (e) to (h) show the same information as (a)-(d), respectively, but for in-plane force excitation of the point source.

To demonstrate the robustness of the topological edge modes, we design a zigzag 
interface separating the two bulk metamaterials $\alpha_{1}$ and $\alpha_{2}$ with the L-L and S-S interfaces respectively. Since typical surface waves such as Rayleigh and Love waves have different polarizations, we study the surface wave-based topological edge modes by using both out-of-plane and in-plane force excitations.

A point source represented by an out-of-plane force (marked as the green star in FIGs. 3(a)-(b)) is excited at the entrance of the zig-zag interface. Low-reflecting boundaries are applied to the surrounding boundaries to eliminate the reflection effects. The edge modes are excited at $10.75 \mathrm{~Hz}$ and $10.40 \mathrm{~Hz}$ and their propagation presented for the L-L (FIG. 3(a)) and S-S (FIG. 3(b)) types interfaces, respectively. In both structures, the wave entering the waveguide travels towards the end and passes the sharp bending corners without a significant backscattering. To further demonstrate that the edge mode is essentially based on surface acoustic waves, we plot the kinetic energy density at the green dots 1 and 2 as a function of the depth in the soil in FIGs. 3(c)-(d) for the two structures. The mode exhibits a high confinement near the surface, with most of the energy localized within one wavelength depth from the ground surface. This behavior supports the conclusion that the designed topological edge states are based on nonleaky surface waves that do not radiate into the bulk. In addition, the energy density at the dot 2 near the exit is a bit smaller than that at the dot 1 near the entrance, remaining at relatively the same level, which also supports high transmission and backscattering immunity of the topological edge mode.

We now perform similar calculations for an excitation by an in-plane point source force with the same amplitude as above. The results are presented in FIGs. 3(e)-(h). The generated surface wave displays a higher weight of the in-plane displacement over total displacement than the case of the out-of-plane force excitation. One can see in FIGs. 3(e)-(f) similar high transmission and backscattering immunity of the topological edge state as above. However, the kinetic energy density (FIGs. 3(g)-(h)) is now smaller than before.

From the dispersion diagram shown in FIG. 2, one can note that the edge mode of the S-S type interface covers a certain frequency range, which makes it a good candidate 

harvesting.

\section{Robustness of the topological edge mode}

(a)

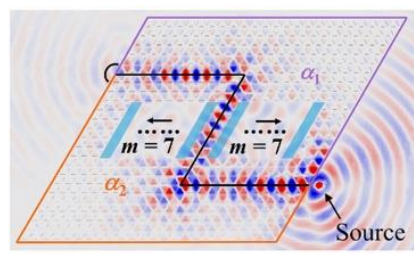

(d)

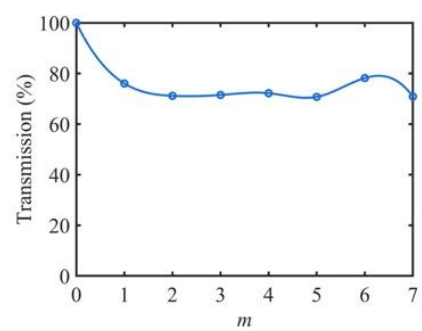

(b)

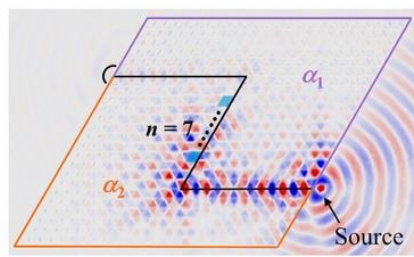

(e)

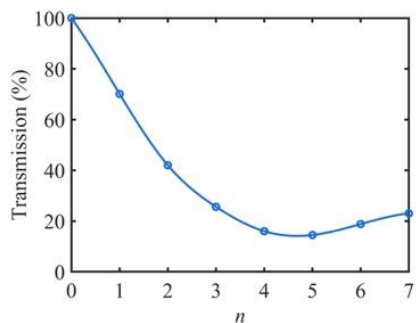

(c)

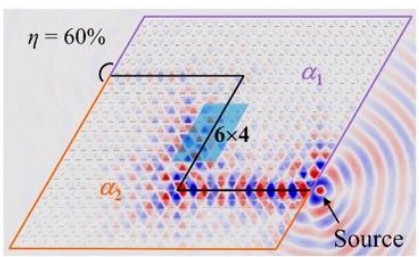

(f)

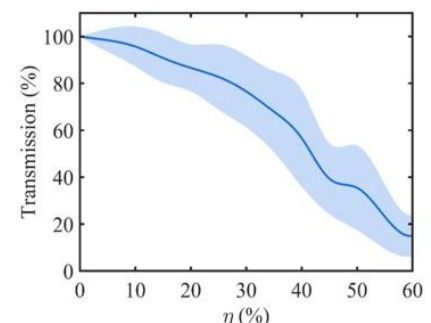

FIG. 4 The robustness of the edge state at the S-S type interface in presence of defects and disorder in height of the pillars at $10.40 \mathrm{~Hz}$. The upper panels show the out-of-plane displacement along the waveguide for a particular choice of disorder whereas the lower panels give the attenuation in the transmission for various degrees of disorder. (a) \& (d) The effect of removing $m$ strips on each side of the interface in the middle part of the waveguide. The blue strip supercell constituting the interface area contains 6 unit cells. The out-of-plane displacement is detected at the black arcs at the exit of the zigzag path. (b) \& (e) The effect of introducing $n$ defects in the unit cells contained in the blue area close to the interface. (c) \& (f) The effect of height disorder by a factor $\eta$ for the 24 units in the blue area. The light blue domain in (f) demonstrates the standard deviation of the transmission for different realizations of the disorder at each $\eta$. In the lower panels, the calculations are performed for discrete values of the abscissa and the continuous lines are a guide for the eyes.

We investigate the robustness of the edge state to propagate along the waveguide, by calculating the loss in the transmission coefficient in presence of a perturbation. Therefore, we define the ratio $\Delta_{1} / \Delta_{2}$, where $\Delta_{1}$ and $\Delta_{2}$ represent the integrated displacements along the green arcs at the exit of the waveguide with and without perturbations, respectively. We consider three kinds of perturbations to quantitatively study the robustness of the topological edge state.

The first perturbation consists of removing $m$ strips on each side of the interface in the middle part of the waveguide as shown in FIG. 4(a). The area delimited by two blue strips on each side of the waveguide is constituted by 6 unit cells. When one strip 
of defect is introduced ( $m=1)$, FIG. 4(d) shows that the transmission decreases suddenly to about $75 \%$; then, by increasing further $m$, it slightly oscillates around the level (70\%-75\%), exhibiting a good robustness to stronger disorder. The displacement field for $m=7$ is calculated and presented in FIG. 4(a), showing a preserved zigzag edge state. Such high robustness is mainly attributed to the strong localization of the topological edge mode in the vicinity of the interface.

The second perturbation (FIGs. 4(b) and (e)) consists of randomly removing $n$ units at the left side of the middle part of the zigzag waveguide. From FIG. 4(e) one can observe that when the defect number $n$ increases from 0 to 4 , the transmission decreases by a large amount to about $20 \%$ of the unperturbed situation, due to the appearance of backscattering effect; then the level of transmission keeps at about $20 \%$ when the defect number continues to increase until 7. The transmission field at $n=7$ is shown in FIG. 4(b) which indicates that although the major part of the wave is reflected, there still remain weak monopolar edge state after the 7-unit-defect along the last horizontal part of the zigzag path, showing a good robustness in edge mode shape.

The third perturbation is dealing with the effect of disorder in the height of a set of 24 pillars located in the blue shaded region of FIG. 4(c). These heights are randomly distributed within the interval $[h-\Delta h, h+\Delta h]$, thus defining a disorder degree $\eta=\Delta h / h$. For a given disorder degree, the average transmission coefficient is evaluated over 30 random samplings and the results are shown as the solid line in FIG. 4(f). We also evaluate the standard deviation of transmissions and plot it as the shaded blue domain around the solid line in FIG. 4(f). The stable level of the standard deviation supports that the random sampling number of 30 should be sufficient. Generally, when the disorder degree $\eta$ increases from 0 to $60 \%$, the average transmission decreases quasilinearly, exhibiting that the backscattering effect also becomes stronger. We show the transmission field at $\eta=60 \%$ in FIG. 4(c). Despite the backscattering effect, the relative weak propagating wave along the last horizontal part of the zigzag path still conserves the monopolar edge state property, showing a strong robustness of the topological edge mode. 


\section{Topological edge mode for energy harvesting}

The robust and compact topological edge mode has a great potential for energy harvesting from a practical point of view. We adopt piezoelectric PZT-5H patches and attach them to the top surface of soil. With the electro-mechanical coupling effect, the vibrating energy of soil can be transformed to electric power. We choose five identical square PZT patches with sides $l=0.1 \mathrm{~m}$ and thicknesses $t=0.001 \mathrm{~m}$, and arrange them alternately as shown by the five yellow squares in FIG. 5(b). A simple circuit powered by each PZT patch with a resistance $R=1000 \Omega$ is devised, whose average output power $P_{i}$ is calculated by

$$
P_{i}=\frac{V_{i}}{\sqrt{2}} \frac{I_{i}}{\sqrt{2}}=\frac{R I_{i}^{2}}{2}
$$

where $V_{i}$ is the output voltage, $I_{i}$ is the loop current to be measured. The detailed

305 information can be found in Appendix B. We sum the total power $P=\sum_{i=1}^{5} P_{i}$ of all the six PZT patches, and present the transmission spectra together with the color information of localization ratio $\beta$ (Eq. (4)) in FIG. 5(a). From FIG. 5(a), the topological edge mode with bright color is successfully captured within the frequency range [10.33, 10.97] $\mathrm{Hz}$ and the peak is measured at $10.40 \mathrm{~Hz}$ which coincides to the design. The electric power at the peak is about two orders of magnitude higher than that within the frequency ra $[9.50,10.00] \mathrm{Hz}$ outside the non-trivial bandgap. Besides, it is more than one order of magnitude higher than that of a bare surface, showing a big advantage of the energy harvesting by topological edge mode. We also present the displacement field at $10.40 \mathrm{~Hz}$ in FIG. 5(b), almost identical to the field without patches (see FIG. 3(b)). In fact, all the vibrating pillars at the interface are available for the energy conversion in practice. Given that the topological edge mode is highly robust to the studied three kinds of perturbations, the corresponding induced energy harvesting also has an efficient robustness.

At the macro scale, the basic functionality of the structure is to robustly isolate surface waves with the non-trivial low-frequency bandgap. Energy harvesting is an 
additional functionality by designing the proper interface path inside the metamaterial.

With maximum vibrating displacements at the interface of the order of $0.5 \mathrm{~mm}$, and the small size of the designed PZT patch in FIG. 5, an electric power of about $10^{-5} \mathrm{~W}$ is achieved. However, if the surface vibrations increase in practice, the PZT patch would be able to generate much higher electric power, making the metamaterial as a potential ground electric generator.

(a)

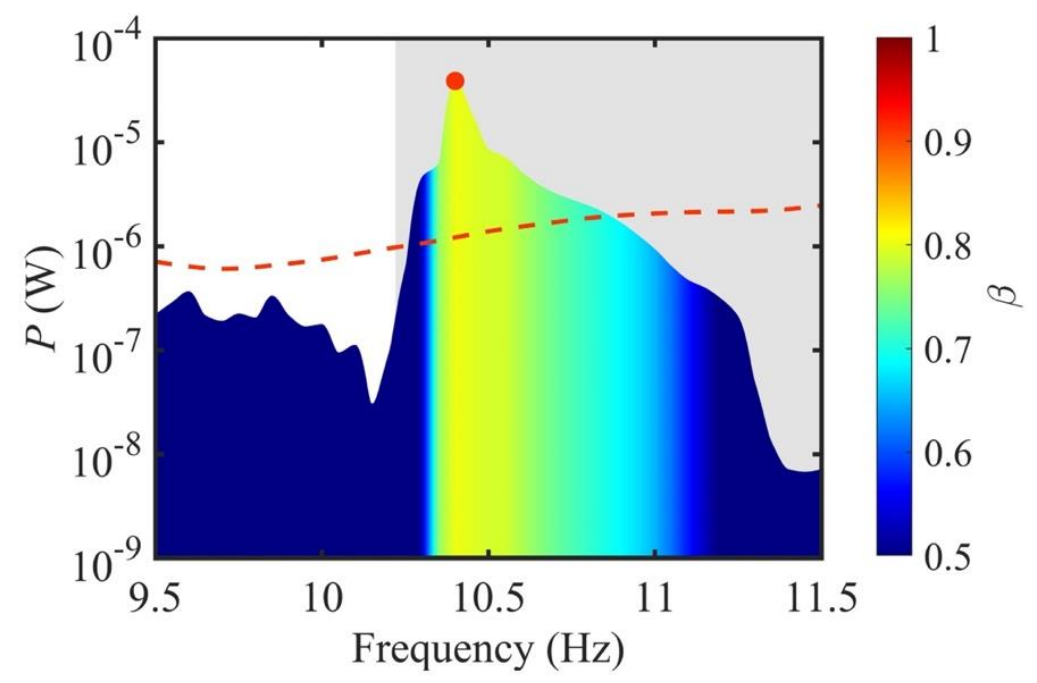

(b)

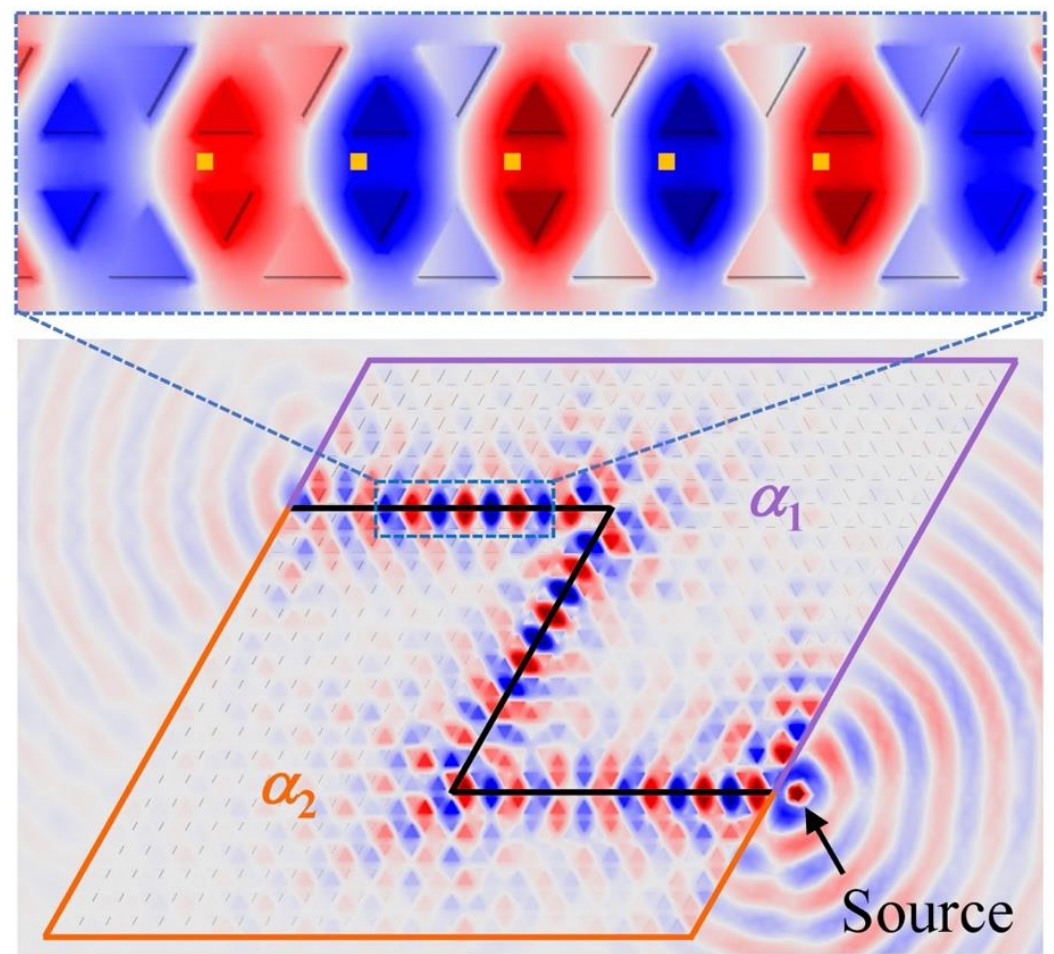

FIG. 5 (a) Piezoelectric energy harvesting for the edge modes with (b) the displacement fields at $10.40 \mathrm{~Hz}$. The red dashed curve in (a) represents the electric power measured without pillars. The 5 
PZT patches are displayed as yellow squares in the zoom-in of (b). The color map in (a) defines the localization ratio $\beta$ defined in Eq. (4).

\section{Conclusion}

A topological surface wave metamaterial composed of triangular section concrete pillars arranged in a honeycomb lattice on the soil is proposed for robust vibration attenuation and energy harvesting at low frequency ranges, below $20 \mathrm{~Hz}$. By breaking the inversion symmetry of the unit cell while preserving $C_{3 \mathrm{v}}$ symmetry, the degeneracy of the Dirac cone at $\mathrm{K}$ point is lifted to open a non-trivial bandgap. By combining two metamaterials with different topological phases, two types of topological edge modes (L-L and S-S types) with backscattering suppression and compact properties can be designed around $10 \mathrm{~Hz}$. We further quantitatively analyzed the robustness of the S-S type topological edge mode against three kinds of perturbations, namely, (i) stripe defects in the vicinity of the interface towards the bulk of the crystal, (ii) unit defects at the interface and (iii) disorders in the height of pillars at the interface. For perturbation (i), the transmission of the topological edge mode keeps stable at relative high level no matter how large is the defect in the bulk media, showing a highly compact property of the topological edge mode. For perturbation (ii), the transmission first decreases very fast before becoming stable when the unit-defect number reaches 4 . For perturbation (iii), the transmission slowly decreases in quasi-linear shape. For the latter two cases, the topological edge modes are still preserved at high considered perturbation degree, exhibiting a good robustness. Finally, piezoelectric patches are designed and attached on top of the pillars at the interface. The robust and compact topological edge mode makes it possible for energy harvesting within the frequency range [10.33, 10.97] Hz of the topological edge mode. The entire size of the pillared metamaterial can be further minimized according to the potential practical demands. For instance, a thickness such as 4 or 5 rows of units is sufficient to effectively attenuate surface waves. The unit cells' geometric parameters can also be optimized for different working frequency range. The employed concrete and PZT are commercial materials with low price, making the metamaterial realizable in practical engineering. The proposed new surface wave 
metamaterial has great potential in robust surface vibration attenuation with the nontrivial low frequency bandgap and robust energy harvesting with the topological edge mode.

\section{Acknowledgment}

This work is supported by the National Natural Science Foundation of China (11902223), the Shanghai Pujiang Program (19PJ1410100), the program for professor of special appointment (Eastern Scholar) at Shanghai Institutions of Higher Learning, the Fundamental Research Funds for the Central Universities, high-level of foreign expert program of Tongji University and Shanghai municipal peak discipline program (2019010106). This work is also partially supported by the French EIPHI Graduate School (contract "ANR-17-EURE-0002").

\section{Data Availability}

The data that support the findings of this study are available from the corresponding author upon reasonable request.

\section{Appendix A. The strip's dispersion calculation}

The strip's dispersion calculation is modeled via the finite element method. In FIG. A1, a stripe consisting of lattices red and blue with an interface at the center is deposited on top of the substrate. The valley Chern number of the two bands associated with the non-trivial bandgap are opposite for the red and blue lattice bulk media. Low reflection boundary is applied to the bottom surface. Periodic conditions are applied to the twoblue side-faces of the substrate along $x$ direction. Stress free conditions are applied to the two-grey side-faces of the substrate along $y$ direction. The built model contains about 350 thousand domain element meshes with about 2.9 million degrees of freedom. 


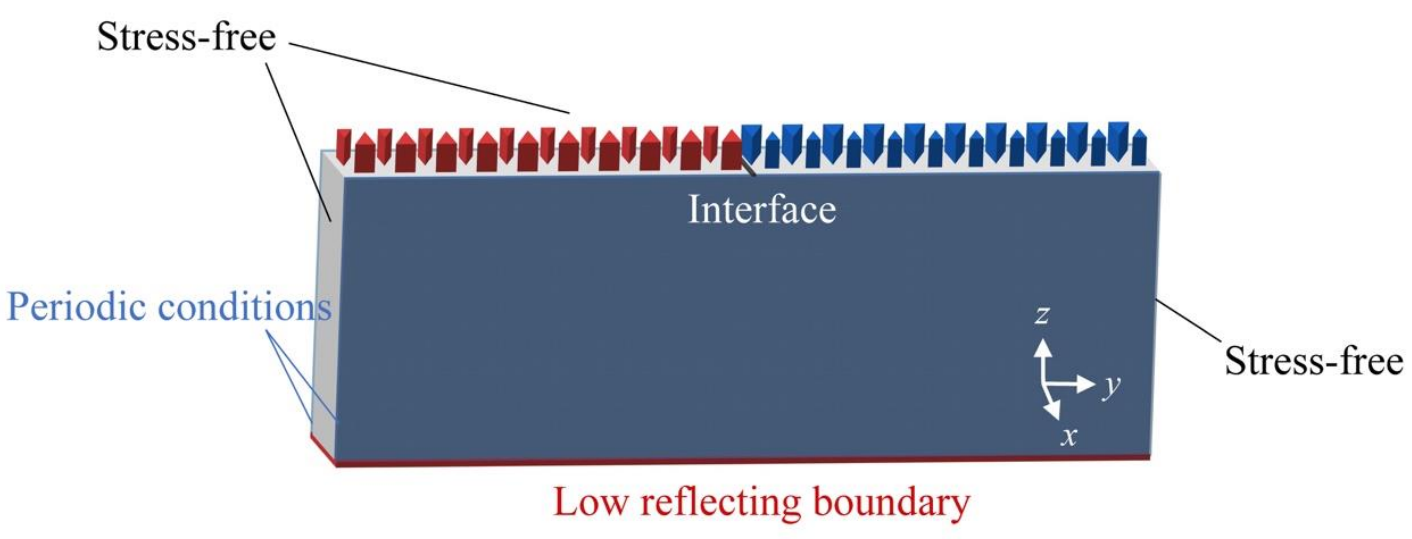

FIG. A1 dispersion model of a stripe waveguide.

\section{Appendix B. Simulating information for the energy harvesting model}

In this section, we detail the simulation process of the transmission with piezoelectric energy harvesting along the zig-zag interface in the COMSOL Multiphysics®. It is calculated by coupling of solid mechanics, electrostatics and electrical circuit. The specific process is (I) Geometry model building; (II) Material and mechanical boundary conditions settings; (III) Piezoelectric and electrical circuit settings; (IV) Meshing; (V) Multiphysics simulation at given frequencies.

To enhance the calculation efficiency, the top surface of this model is discretized by free triangular elements, and the entire geometry is swept with appropriate element size. The meshed model contains 1330471 domain elements and 7879642 degrees of freedom, which is sufficient to ensure the convergence of the results. For calculation stability, we use the CPU of Intel® Xeon ${ }^{\circledR}$ Gold 6154 Processor with the max turbo frequency of $3.70 \mathrm{GHz}$ and the memory of $512 \mathrm{~GB}$. The simulation costs about one and a half hours for each frequency.

\section{References}

[1] M. Miniaci, A. Krushynska, F. Bosia, N.M. Pugno, Large scale mechanical metamaterials as seismic shields, New Journal of Physics, 18 (2016) 083041.

[2] R. Cai, Y. Jin, T. Rabczuk, X. Zhuang, B. Djafari-Rouhani, Propagation and attenuation of Rayleigh and pseudo surface waves in viscoelastic metamaterials, Journal of Applied Physics, 129 (2021) 124903. [3] Y. Jin, Y. Pennec, B. Bonello, H. Honarvar, L. Dobrzynski, B. Djafari-Rouhani, M. Hussein, Physics of surface vibrational resonances: Pillared phononic crystals, metamaterials, and metasurfaces, Rep Prog Phys, DOI 10.1088/1361-6633/abdab8(2021). 

214303.

[5] Z. Liu, X. Zhang, Y. Mao, Y.Y. Zhu, Z. Yang, C.T. Chan, P. Sheng, Locally Resonant Sonic Materials, Science, 289 (2000) 1734.

[6] Y. Jin, N. Fernez, Y. Pennec, B. Bonello, R.P. Moiseyenko, S. Hémon, Y. Pan, B. Djafari-Rouhani,

Tunable waveguide and cavity in a phononic crystal plate by controlling whispering-gallery modes in hollow pillars, Physical Review B, 93 (2016) 054109

[7] M. Oudich, B. Djafari-Rouhani, B. Bonello, Y. Pennec, S. Hemaidia, F. Sarry, D. Beyssen, Rayleigh

421

422

423

424

425

426

427

428

429

430

431

432

433

434

435

436

437

438

439

440

441

442

443

444

445

446

447

448

449

450

451

452

453

454

455

Waves in Phononic Crystal Made of Multilayered Pillars: Confined Modes, Fano Resonances, and Acoustically Induced Transparency, Physical Review Applied, 9 (2018) 034013.

[8] Y. Chen, L. Wang, Isolation of Surface Wave-Induced Vibration Using Periodically Modulated Piles, International Journal of Applied Mechanics, 06 (2014) 1450042.

[9] Y. Achaoui, B. Ungureanu, S. Enoch, S. Brûlé, S. Guenneau, Seismic waves damping with arrays of inertial resonators, Extreme Mechanics Letters, 8 (2016) 30-37.

[10] X. Cai, L. Ye, C. Qiu, M. Xiao, R. Yu, M. Ke, Z. Liu, Symmetry-enforced three-dimensional Dirac phononic crystals, Light Sci Appl, 9 (2020) 38.

[11] R. Chaunsali, C.-W. Chen, J. Yang, Subwavelength and directional control of flexural waves in zonefolding induced topological plates, Physical Review B, 97 (2018) 054307.

[12] G. Ma, M. Xiao, C.T. Chan, Topological phases in acoustic and mechanical systems, Nature Reviews Physics, 1 (2019) 281-294.

[13] B.H. Nguyen, H.S. Park, X. Zhuang, T. Rabczuk, Tunable topological bandgaps and frequencies in a pre-stressed soft phononic crystal, Journal of Applied Physics, 125 (2019) 095106.

[14] R. Chaunsali, C.-W. Chen, J. Yang, Experimental demonstration of topological waveguiding in elastic plates with local resonators, New Journal of Physics, 20 (2018) 113036.

[15] H. Fan, B. Xia, L. Tong, S. Zheng, D. Yu, Elastic Higher-Order Topological Insulator with Topologically Protected Corner States, Physical Review Letters, 122 (2019) 204301.

[16] Y. Jin, W. Wang, Z. Wen, D. Torrent, B. Djafari-Rouhani, Topological states in twisted pillared phononic plates, Extreme Mechanics Letters, 39 (2020) 100777.

[17] J. Chen, H. Huang, S. Huo, Z. Tan, X. Xie, J. Cheng, G.-l. Huang, Self-ordering induces multiple topological transitions for in-plane bulk waves in solid phononic crystals, Physical Review B, 98 (2018) 014302 .

[18] H. Huang, Z. Tan, S. Huo, L. Feng, J. Chen, X. Han, Topologically protected zero refraction of elastic waves in pseudospin-Hall phononic crystals, Communications Physics, 3 (2020) 46.

[19] H. Huang, S. Huo, J. Chen, Subwavelength elastic topological negative refraction in ternary locally resonant phononic crystals, International Journal of Mechanical Sciences, 198 (2021) 106391.

[20] W. Wang, Y. Jin, W. Wang, B. Bonello, B. Djafari-Rouhani, R. Fleury, Robust Fano resonance in a topological mechanical beam, Physical Review B, 101 (2020).

[21] C. Goffaux, J. Sánchez-Dehesa, A.L. Yeyati, P. Lambin, A. Khelif, J.O. Vasseur, B. Djafari-Rouhani, Evidence of Fano-Like Interference Phenomena in Locally Resonant Materials, Physical Review Letters, 88 (2002) 225502.

[22] Y. Jin, Y. Pennec, B. Djafari-Rouhani, Acoustic analogue of electromagnetically induced transparency and Autler-Townes splitting in pillared metasurfaces, Journal of Physics D: Applied Physics, 51 (2018) 494004. 
[23] Y. Jin, E.I.H. Ei Boudouti, Y. Pennec, B. Djafari-Rouhani, Tunable Fano resonances of Lamb modes in a pillared metasurface, Journal of Physics D: Applied Physics, 50 (2017).

[24] Z. Wen, S. Zeng, D. Wang, Y. Jin, B. Djafari-Rouhani, Robust edge states of subwavelength chiral phononic plates, Extreme Mechanics Letters, DOI 10.1016/j.eml.2021.101209(2021).

[25] Y. Jin, W. Wang, A. Khelif, B. Djafari-Rouhani, Elastic Metasurfaces for Deep and Robust Subwavelength Focusing and Imaging, Physical Review Applied, 15 (2021).

[26] S. Brûlé, S. Enoch, S. Guenneau, Emergence of seismic metamaterials: Current state and future perspectives, Physics Letters A, 384 (2020) 126034.

[27] A. Colombi, D. Colquitt, P. Roux, S. Guenneau, R.V. Craster, A seismic metamaterial: The resonant metawedge, Sci Rep, 6 (2016) 27717.

[28] A. Colombi, P. Roux, S. Guenneau, P. Gueguen, R.V. Craster, Forests as a natural seismic metamaterial: Rayleigh wave bandgaps induced by local resonances, Sci Rep, 6 (2016) 19238.

[29] T. Li, Q. Su, S. Kaewunruen, Seismic metamaterial barriers for ground vibration mitigation in railways considering the train-track-soil dynamic interactions, Construction and Building Materials, 260 (2020) 119936.

[30] D. Mu, H. Shu, L. Zhao, S. An, A Review of Research on Seismic Metamaterials, Advanced Engineering Materials, 22 (2020) 1901148.

[31] Muhammad, C.W. Lim, J.N. Reddy, Built-up structural steel sections as seismic metamaterials for surface wave attenuation with low frequency wide bandgap in layered soil medium, Engineering Structures, 188 (2019) 440-451.

[32] X. Pu, A. Palermo, Z. Cheng, Z. Shi, A. Marzani, Seismic metasurfaces on porous layered media: Surface resonators and fluid-solid interaction effects on the propagation of Rayleigh waves, International Journal of Engineering Science, 154 (2020) 103347.

[33] P.T. Wootton, J. Kaplunov, D.J. Colquitt, An asymptotic hyperbolic-elliptic model for flexuralseismic metasurfaces, Proc Math Phys Eng Sci, 475 (2019) 20190079.

[34] Y. Zeng, Y. Xu, K. Deng, Z. Zeng, H. Yang, M. Muzamil, Q. Du, Low-frequency broadband seismic metamaterial using I-shaped pillars in a half-space, Journal of Applied Physics, 123 (2018) 214901.

[35] W. Liu, G.H. Yoon, B. Yi, Y. Yang, Y. Chen, Ultra-wide band gap metasurfaces for controlling seismic surface waves, Extreme Mechanics Letters, 41 (2020).

[36] Y. Zeng, P. Peng, Q.-J. Du, Y.-S. Wang, B. Assouar, Subwavelength seismic metamaterial with an ultra-low frequency bandgap, Journal of Applied Physics, 128 (2020) 014901.

[37] D. Torrent, D. Mayou, J. Sánchez-Dehesa, Elastic analog of graphene: Dirac cones and edge states for flexural waves in thin plates, Physical Review B, 87 (2013) 115143.

[38] G.J. Chaplain, J.M.D. Ponti, G. Aguzzid, A. Colombid, R.V. Craster, Topological Rainbow Trapping for Elastic Energy Harvesting in Graded Su-Schrieffer-Heeger Systems, Physical Review Applied, 14 (2020) 054035.

[39] J.-J. Chen, S.-Y. Huo, Z.-G. Geng, H.-B. Huang, X.-F. Zhu, Topological valley transport of platemode waves in a homogenous thin plate with periodic stubbed surface, AIP Advances, 7 (2017).

[40] Y. Jin, D. Torrent, B. Djafari-Rouhani, Robustness of conventional and topologically protected edge states in phononic crystal plates, Physical Review B, 98 (2018).

[41] R.K. Pal, M. Ruzzene, Edge waves in plates with resonators: an elastic analogue of the quantum valley Hall effect, New Journal of Physics, 19 (2017) 025001.

[42] J. Vila, R.K. Pal, M. Ruzzene, Observation of topological valley modes in an elastic hexagonal lattice, Physical Review B, 96 (2017) 134307. 
[43] M. Yan, J. Lu, F. Li, W. Deng, X. Huang, J. Ma, Z. Liu, On-chip valley topological materials for

501 elastic wave manipulation, Nat Mater, 17 (2018) 993-998.

502 [44] Z. Wang, F.-K. Liu, S.-Y. Yu, S.-L. Yan, M.-H. Lu, Y. Jing, Y.-F. Chen, Guiding robust valley503 dependent edge states by surface acoustic waves, Journal of Applied Physics, 125 (2019) 044502.

504 [45] T. Inoue, S. Murakami, Topological band structure of surface acoustic waves on a periodically 505 corrugated surface, Physical Review B, 99 (2019) 195443.

506 [46] D.R. Hofstadter, Energy levels and wave functions of Bloch electrons in rational and irrational 507 magnetic fields, Physical Review B, 14 (1976) 2239-2249.

508 [47] B. Graczykowski, F. Alzina, J. Gomis-Bresco, C.M. Sotomayor Torres, Finite element analysis of 509 true and pseudo surface acoustic waves in one-dimensional phononic crystals, Journal of Applied 510 Physics, 119 (2016) 025308.

511 [48] L. He, Z. Wen, Y. Jin, D. Torrent, X. Zhuang, T. Rabczuk, Inverse design of topological metaplates 512 for flexural waves with machine learning, Materials \& Design, 199 (2021) 109390. 